\title{
Whole blood microarray analysis of pigs showing extreme phenotypes after a porcine reproductive and respiratory syndrome virus infection
}

\author{
Martine Schroyen ${ }^{1}$, Juan P. Steibel ${ }^{2,3^{*}}$, James E. Koltes ${ }^{1}$, Igseo Choi ${ }^{4}$, Nancy E. Raney ${ }^{2}$, Christopher Eisley ${ }^{5}$, \\ Eric Fritz-Waters', James M. Reecy ${ }^{1}$, Jack C. M. Dekkers ${ }^{1}$, Robert R. R. Rowland ${ }^{6}$, Joan K. Lunney ${ }^{4}$, \\ Catherine W. Ernst ${ }^{2}$ and Christopher K. Tuggle ${ }^{1 *}$
}

\begin{abstract}
Background: The presence of variability in the response of pigs to Porcine Reproductive and Respiratory Syndrome virus (PRRSv) infection, and recent demonstration of significant genetic control of such responses, leads us to believe that selection towards more disease resistant pigs could be a valid strategy to reduce its economic impact on the swine industry. To find underlying molecular differences in PRRS susceptible versus more resistant pigs, 100 animals with extremely different growth rates and viremia levels after PRRSv infection were selected from a total of 600 infected pigs. A microarray experiment was conducted on whole blood RNA samples taken at 0, 4 and 7 days post infection (dpi) from these pigs. From these data, we examined associations of gene expression with weight gain and viral load phenotypes. The single nucleotide polymorphism (SNP) marker WUR10000125 (WUR) on the porcine $60 \mathrm{~K} \mathrm{SNP}$ chip was shown to be associated with viral load and weight gain after PRRSv infection, and so the effect of the WUR10000125 (WUR) genotype on expression in whole blood was also examined.

Results: Limited information was obtained through linear modeling of blood gene differential expression (DE) that contrasted pigs with extreme phenotypes, for growth or viral load or between animals with different WUR genotype. However, using network-based approaches, molecular pathway differences between extreme phenotypic classes could be identified. Several gene clusters of interest were found when Weighted Gene Co-expression Network Analysis (WGCNA) was applied to 4dpi contrasted with Odpi data. The expression pattern of one such cluster of genes correlated with weight gain and WUR genotype, contained numerous immune response genes such as cytokines, chemokines, interferon type I stimulated genes, apoptotic genes and genes regulating complement activation. In addition, Partial Correlation and Information Theory (PCIT) identified differentially hubbed (DH) genes between the phenotypically divergent groups. $\mathrm{GO}$ enrichment revealed that the target genes of these $\mathrm{DH}$ genes are enriched in adaptive immune pathways.
\end{abstract}

Conclusion: There are molecular differences in blood RNA patterns between pigs with extreme phenotypes or with a different WUR genotype in early responses to PRRSV infection, though they can be quite subtle and more difficult to discover with conventional DE expression analyses. Co-expression analyses such as WGCNA and PCIT can be used to reveal network differences between such extreme response groups.

Keywords: Pig, PRRS, Microarray, Transcriptomics, WGCNA, PCIT, Immune response

\footnotetext{
* Correspondence: steibelj@msu.edu; cktuggle@iastate.edu

${ }^{2}$ Department of Animal Science, Michigan State University, East Lansing, MI,

USA

'Department of Animal Science, lowa State University, Ames, IA, USA

Full list of author information is available at the end of the article
} 


\section{Background}

In the United States, Porcine Reproductive and Respiratory Syndrome (PRRS) is one of the most economically devastating diseases currently in the swine industry $[1,2]$. PRRS affects all production stages, manifesting reproductive losses (infertility, abortions, and stillborn and mummified fetuses), and piglets show a higher pre-weaning morbidity and mortality rate, persisting with a reduced thrift throughout the entire grow-finishing period [3]. The disease is caused by the PRRS virus (PRRSv), an enveloped, single stranded RNA virus that belongs to the Arteriviridae family $[4,5]$. PRRSv uses complex strategies to evade both the innate and adaptive immune responses [6]. Because these immune evasion mechanisms are not fully understood, a sustainable treatment is difficult to find. The ease with which PRRSv moves from farm to farm further complicates control strategies [7]. In addition, the virus is genetically highly heterologous and vaccination based on a single PRRSv strain is not necessarily sufficient to protect against other strains [6].

One way to minimize the economic loss caused by PRRS is to improve disease resistance of the host. The PRRS Host Genetics Consortium (PHGC) was founded to examine the genetic basis of host responses to PRRS and understand its overall impact on pig health and growth [8]. As part of the PHGC, infection trials are conducted on approximately 200 weanling pigs each. All pigs in these studies are infected with PRRSv, and weight gain and viremia levels are measured on $0,4,7,11,14$, $19 / 21,28,35$ days post infection (dpi) to day 40/42, when the trial is terminated. In 2012, using a genomewide association study on the first three infection trials, Boddicker et al. [9] reported a quantitative trait locus (QTL) on chromosome 4 (SSC4) that explained a large proportion of the genetic variance for viral load and, to a lesser extent, weight gain. In that region on SSC4, the single nucleotide polymorphism (SNP) marker WUR10000125 (WUR) on the porcine $60 \mathrm{~K} \mathrm{SNP} \mathrm{chip}$ was shown to capture most of the effect in this region. The effect of the SSC4 region, and of WUR in particular, was successfully validated in additional trials on animals with a different genetic background [10, 11]. For WUR, the $\mathrm{B}$ allele is the favorable allele when compared to the A allele, but the B allele has a low frequency in these challenge populations. Fortunately, the SNP marker works in a dominant manner, giving similar protective phenotypes for $\mathrm{AB}$ and $\mathrm{BB}$ animals [9].

In the last couple of years, several porcine gene expression studies, primarily at the cell culture infection level, have been executed in an attempt to unravel the porcine immune responses evoked by the PRRS virus. Of these whole genome PRRS expression studies, some focused on expression differences as response to virus strains with different pathogenicities [12, 13], others calculated expression differences between infected and control (uninfected) pigs [14-16] or in vitro between infected and control cells [17], and two measured whole genome expression differences due to breed [18, 19]. These latter experiments compared breeds that are more resistant to PRRS with breeds that are more susceptible to it, and were focused on understanding immunological differences to explain phenotypic differences. Thus, these experiments reported on gene expression in dissected tissue, a method that would be difficult and costly to implement in a practical selection process. In the current PHGC gene expression study, all animals were infected and comparisons were made between susceptible and more resistant pigs within breed. The difference in susceptibility was indirectly measured by growth rate post-infection and viremia levels in the blood. Bates et al. [20] earlier reported a similar study using infected susceptible pigs, that showed a high PRRSv burden (high responders, $\mathrm{H}$ ) and infected but tolerant or resistant animals with a low PRRSv burden (low responders, L). At $14 \mathrm{dpi}$, lungs and bronchial lymph nodes were collected and several genes such as CCAAT/enhancer-binding $\delta$ protein (CEBP $\delta$ ) and thioredoxin-interacting protein (TXNIP), with a differential expression (DE) level between $\mathrm{H}$ and $\mathrm{L}$ in one or both tissues, were found using the $13 \mathrm{~K}$ Qiagen-NRSP8 porcine oligo array [20]. A follow-up study using the $20 \mathrm{~K}$ Pigoligoarray on the same samples confirmed the DE of several of these candidate genes, as well as additional immune response candidate genes [21].

In gene expression studies conducted on pigs within the PHGC trials, the blood transcriptome is examined because of its collection ease, the large number of animals that can be sampled, the possibility of repeated sampling of the same individual, and, ultimately, the chance to develop biomarkers for selection purposes. In 2013, an initial gene expression study performed on the first PHGC trial was published [22]. In this study, twelve animals that represented all four combinations of two extreme phenotypes regarding weight gain (high growth rate versus low growth rate) and viral load (high viral load versus low viral load) were selected for study. The blood transcriptome of these twelve pigs on several dpi was compared using the Pigoligoarray [23], the annotation used was the current NCBI RefSeq annotation. Array probes and their annotation can be downloaded from www.animalgenome.org/pig/ projects/oligoAnnot/2014/ (see GPL7435 array). One main goal of the Arceo et al. [22] study was to determine an adequate sample size and to decide which dpi were the most informative with regard to future PRRS response expression studies in the blood. The study described herein is an expansion to 
that study, investigating the expression profile of sufficient numbers of blood samples as proposed by Arceo et al. [22]. Samples were taken at three early time points $(0,4$ and $7 \mathrm{dpi})$ for at least 20 infected animals per phenotypic group. A linear modeling approach was used to find DE genes between phenotypic extremes or different WUR genotypes. Besides annotation analyses of DE gene lists, weighted gene co-expression network analyses (WGCNA) [24] and partial correlation and information theory (PCIT) [25] were used to explore these expression datasets. Co-expression changes that look at how clustering or correlations change in response to treatment are more sensitive to detect pathway signaling differences between such treatments. In this way, coexpression analyses may be more sensitive at detecting biologically interesting effects than differential expression analyses. The WGCNA and PCIT approaches allow us to go beyond lists of individual DE genes and identify gene expression networks correlated with relevant phenotypes or WUR genotype.

\section{Methods}

\section{Study design and phenotypic groups}

This study was conducted as part of the PHGC project. Experimental design, details of the infection and tissue collection procedures are described in Lunney et al. [8] and Rowland et al. [26]. Briefly, in each PHGC trial, approximately 200 pigs were transported at weaning age to the biosecure testing facility at Kansas State University and allocated in pens of 10 to 15 pigs per pen. All animals came from PRRSv, Mycoplasma hyopneumoniae and swine influenza virus free high health farms. After a one-week acclimation, pigs were intramuscularly and intranasally infected with a known isolate of PRRSv $\left(10^{5}\right.$ TCID $_{50}$ of NVSL 97-7985). Blood samples were taken at 0 , 4, 7, 11, 14, 19/21, 28, 35 and 40/42 dpi. Viremia levels on these dpi were measured in the serum using qRT-PCR, as described by Boddicker et al. [9]. Weight was measured at day 0 and weekly thereafter. Pigs were euthanized at $42 \mathrm{dpi}$. Viral load (VL) was measured as area under the log curve of these viremia levels from 0 to $21 \mathrm{dpi}$. Weight gain (WG) was measured from 0 to $42 \mathrm{dpi}$. Animals used in this study were part of trials 1,3 and 4. Pigs used in PHGC trial 1 (PHGC1) and PHGC3 were the offspring of Landrace boars and Large White sows, and the average viremia levels on 4, 7, 11, 14 and $21 \mathrm{dpi}$, VL over 21dpi and WG over 21 and 42dpi of these animals can be found in Boddicker et al. [9]. Pigs in PHGC4 came from Duroc sires crossed with Large White/Landrace/Yorkshire sows. Additional details on VL and WG for these animals is reported in
Boddicker et al. [10]. Animals were assigned to four phenotypic groups according to VL and WG, as described by Arceo et al. [22], with minor variation: the criteria for selection was that the normalized weight and viral load were larger than 0.25 standard deviations (SD) of the mean instead of 0.5 SD of the mean. The groups were defined as follows: high VL with maximal WG, referred to as $\mathrm{HvHg}$; high $\mathrm{VL}$ with reduced WG or HvLg; low VL with maximal WG, or LvHg; and low VL with reduced WG, or LvLg (Fig. 1). An overview of the animals used is given in Table 1. Besides collecting blood for viremia measurements, $3 \mathrm{~mL}$ of blood samples were also collected into Tempus ${ }^{\text {Tix }}$ Blood RNA tubes (Life Technologies, Carlsbad, CA, USA) at 0,4 , and $7 \mathrm{dpi}$. Total RNA was extracted using Tempus ${ }^{\text {Tu }}$ Spin RNA Isolation Kit (Life Technologies, Carlsbad, CA, USA) according to the manufacturer's protocol. RNA concentration was quantified using a NanoDrop ND1000 spectrophotometer (Nano-Drop Technologies, Wilmington, DE, USA) and RNA quality was assessed using an Agilent Bioanalyzer 2100 (Agilent Technologies, Inc., Santa Clara, CA, USA).

\section{Ethical statement}

The study was approved by the Kansas State University Institutional Animal Care and Use Committee (IACUC).

\section{Microarray design and analysis}

RNA samples $(0,4$ and $7 \mathrm{dpi})$ were reverse transcribed using the Amino Allyl MessageAmp II aRNA Amplification Kit (Ambion/Life Technologies), labeled with $\mathrm{N}$-hydroxysuccinate (NHS) ester Cy3 or Cy5 dyes (GE Healthcare, CA, USA), and hybridized to a previously described $20 \mathrm{~K} 70$-mer oligonucleotide microarray, named the Pigoligoarray [23]. A block reference design [27] was followed to allocate samples to slides with each individual pig's 0dpisample serving as reference for the other two samples from the same animal. Reference sample dye flipping was performed across replicates within phenotypic groups to allow separation of dye and Odpi effects [27]. Fluorescent images and fluorescence intensity data were collected as previously described [22, 23]. Median intensities were background corrected with Normexp method fixing the offset parameter $\kappa=50$ [28]. Background corrected data was normalized using a within print-tip loesslocation normalization [29]. All computations were implemented in $\mathrm{R}$ [30] through LIMMA [31]. Normalized log-ratios of $4 \mathrm{dpi}-0 \mathrm{dpi}$ and $7 \mathrm{dpi}-0 \mathrm{dpi}$ were analyzed separately. A linear model, accounting for dye, array, trial, group and WUR SNP genotype was fit on a spot oligonucleotide basis [32] using the 


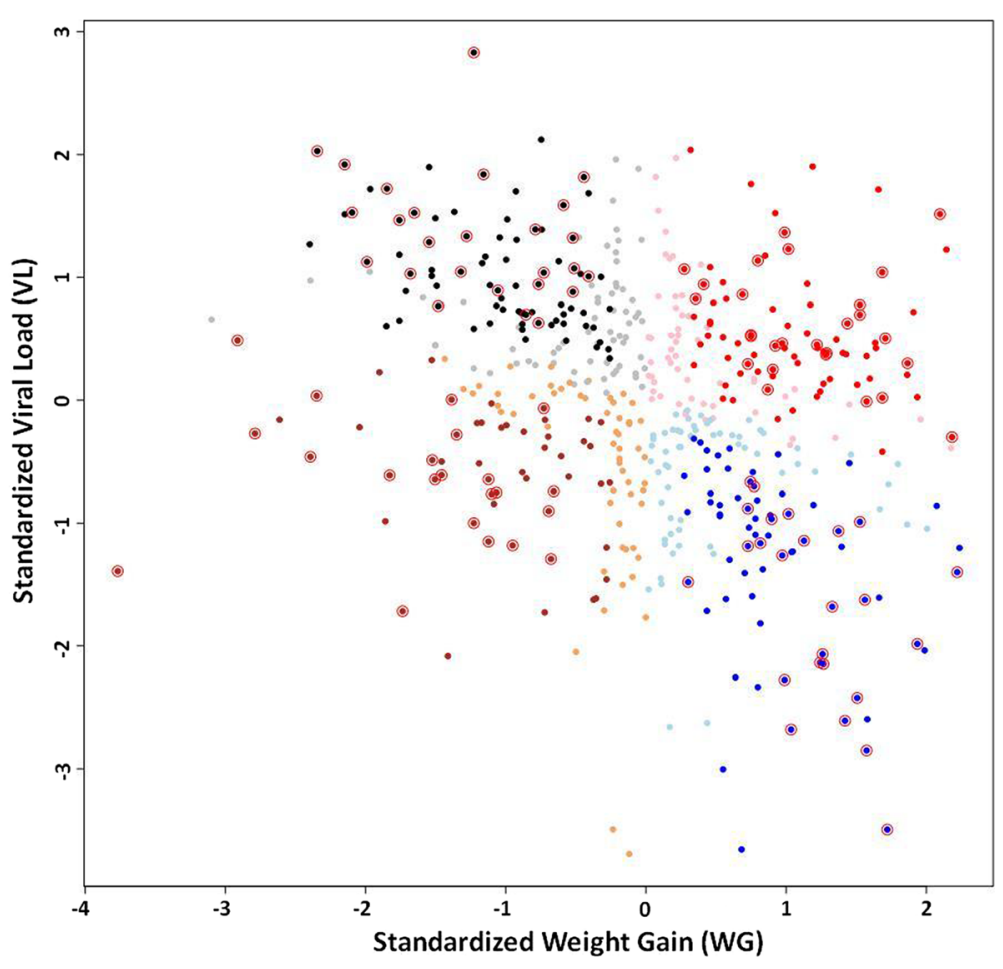

Fig. 1 Scatterplot of PHGC1, 3, 4 animal phenotypes as shown as a function of WG and VL. Each symbol represents a pig. There are 598 pigs in total. The four phenotypic groups ( $\mathrm{LvHg}, \mathrm{HvHg}$, LVLg and HvLg) are represented by a different color. The values for WG and VL are the residuals after correction for trial. The extreme animals for each group are marked with a dark color. The 100 animals selected for the microarray study are marked by circles

LIMMA program [31]. Several contrasts were computed: 1) WUR genotypes, 2) interaction between growth and viral load groups, 3) effect of viral load within growth group, 4) effect of growth within viral load group and 5) $4 \mathrm{dpi}$ or $7 \mathrm{dpi}$ versus $0 \mathrm{dpi}$. To account for multiple testing, the false discovery rate (FDR: q-value) procedure [33] was used to adjust pvalues obtained for each contrast.

\section{Validation of the microarray results using RNAseq}

Validation of DE gene lists of genes with a $q$-value $\leq 0.05$ was done using log normalized and model adjusted expression values obtained from an RNAseq experiment $[34,35]$. In short, the RNAseq experiment was

Table 1 Number of animals used in this study and their phenotypes

\begin{tabular}{|c|c|c|c|c|c|c|c|}
\hline \multirow[t]{2}{*}{ Trial } & \multicolumn{4}{|c|}{ \# of animals per group } & \multicolumn{3}{|c|}{ \# of animals per WUR genotype } \\
\hline & $\mathrm{HvHg}$ & HvLg & $\mathrm{LvHg}$ & $\operatorname{LvLg}$ & $\mathrm{AA}$ & $A B$ & BB \\
\hline PHGC 1 & 10 & 10 & 9 & 8 & 26 & 11 & 0 \\
\hline PHGC 3 & 9 & 9 & 10 & 8 & 21 & 14 & 1 \\
\hline PHGC 4 & 8 & 7 & 6 & 6 & 24 & 3 & 0 \\
\hline Total & 27 & 26 & 25 & 22 & 71 & 28 & 1 \\
\hline
\end{tabular}

Numbers are given for each PHGC trial separately. Groups are formed according to viral load and weight gain $(\mathrm{HvHg}, \mathrm{HvLg}, \mathrm{LvHg}$ and $\mathrm{LvLg})$; genotypes are $A A, A B$ and $B B$ for the WUR10000125 SNP marker performed on 16 animals of PHGC3, 7 of which were among the 100 animals used in the microarray experiment, the expression profiles of the remaining 9 animals are independent. To normalize the RNAseq data, the Trimmed Mean of M-values (TMM) method was used (edgeR package version 3.4.2). Data for lowly expressed genes, whose maximum TMM expression value across samples was less than 10, were removed, and model adjustments were made for pre- and post-globin reduction RNA Integrity Number, and 5' -3 ' read skewness. In all, 8,997 annotated genes were retained. For the 4dpi-0dpi microarray dataset, 31 of the 67 genes from the DE list at a q-value of $\leq 0.05$ were among those 8,997 genes. For the $7 \mathrm{dpi}$-0dpi, 15 of 34 were annotated in both the microarray and RNAseq experiment. Those common 31 and 15 genes were used to test validation of the $4 \mathrm{dpi}$ 0dpi and 7dpi-0dpi microarray experiment, respectively.

\section{WGCNA analysis and module stability}

The WGCNA R package was used to cluster highly correlated genes and find clusters whose expression was correlated with the traits examined [24]. WGCNA was carried out on data from all 19,981 oligonucleotides of the 4dpi-0dpi dataset for all 100 animals. An adjacency matrix based on expression correlation was created using a soft threshold procedure to allow a scale free 
topology. The clusters created by WGCNA are called modules, and the minimum number of genes in a module was set to 30 . Genes not classified in a correlated module were grouped in a grey 'rest of data' module. To see whether modules were stable for each dataset, the module stability was examined using the module stability analysis embedded in the WGCNA package [36].

Once the modules were created, the animals' phenotypic information was correlated with the module eigengene (ME). The eigengene of a module is defined as the eigenvector associated with the first principal component of the expression matrix and is used as a 'supergene' or a linear combination of expression from all genes in the module [37]. Phenotypes to analyze in our experiments were WG, VL and WUR genotype. For WG and $\mathrm{VL}$, the raw values were adjusted for trial mean effects by computing the residuals of a linear model that included the categorical effect of trial. These adjusted values will be referred to as WG residual and VL residual. Because the desired values of WG (higher) and VL (lower) are opposite in direction, a Desirability coefficient (Des coef) was calculated to combine the WG and VL variables as follows: $\frac{W G-\overline{W G}}{\operatorname{stdev}(W G)}-\frac{V L-\overline{V L}}{\operatorname{stdev}(V L)}$. The WUR genotype was coded so that $\mathrm{BB}$ was $-1, \mathrm{AB}$ was 0 and AA was +1 . Additionally, correlations were calculated between the MEs and weight and viremia in the serum on specific days. For weight, this was done at $0,7,14$, $19 / 21,28,35$ and $40 / 42 \mathrm{dpi}$, for viremia days examined were $4,7,11,14$ and 19/21 dpi.

\section{CTEN analysis}

Cell type enrichment (CTEN) was used to see if WGCNA modules that were significantly correlated with a trait of interest, pointed to enrichment of one or more specific cell types to explain expression patterns of specific modules [38]. For this analysis, all available gene symbols in a module were uploaded and the list was compared to the existing CTEN database [38], which consists of highly expressed cell specific genes known for human and mouse. As output, the program uses Benjamini-Hochberg adjusted p-values to determine the significance of enriched cell types or tissues and creates color-coded figures indicating this enrichment.

\section{PCIT analysis}

To identify potential different regulators in phenotypically divergent animals, the PCIT algorithm was run. Full details of this algorithm are described in Reverter and Chen [25] and in Koesterke et al. [39, 40]. In this study, pairwise contrasts were made between $\mathrm{Hg}$ and $\mathrm{Lg}$ animals, between $\mathrm{Hv}$ and $\mathrm{Lv}$ animals, between High Des coef (higher WG and lower VL) and Low Des coef animals, and between animals with a AA versus AB WUR genotype. Since there was only one BB animal, it was omitted in this analysis. The significance of a partial correlation between a target and hub gene was determined using an information theory approach that sets the significance threshold based on the direct and partial correlation for all tests performed in the data [25]. In this way, the significance of an edge in the network is determined by the information in a specific dataset. Only significant partial correlations were used in the differentially hubbed $(\mathrm{DH})$ gene analysis. A script was written to determine the $\mathrm{DH}$ results that identified the hub genes [40].

\section{GO Term Enrichment}

Throughout the experiment, the functional annotation tool DAVID Bioinformatic Resources v6.7 [41] was used to define gene ontology terms enriched by a set of genes. First, DAVID analyses were performed on DE lists created using a maximum FDR adjusted $p$-value of 0.10 as criterion [33]. Second, DAVID analyses were performed on lists of genes corresponding to significant WGCNA modules. WGCNA modules were considered significant for a certain trait when the nominal $p$-value of the correlation between the ME and the trait of interest was less than 0.10. Third, DAVID analyses were performed on the entire list of differentially wired correlates from the top 10 hub genes resulting from the PCIT analysis. Annotations were performed using the human Ensembl gene ID numbers, to maximize recognition by the DAVID tool. As a background gene dataset for these DAVID analyses, all annotated genes of the microarray were used. Enrichment scores higher than 1.3 were regarded as significant.

\section{Results}

The use of microarrays to analyze expression differences due to infection, between phenotypic groups and between animals having a different WUR genotype

To determine the gene expression differences among the four extreme growth and viremia phenotypes in response to PRRSv infection, we selected $\sim 25$ pigs that belonged to each of the $\mathrm{HvHg}, \mathrm{HvLg}, \mathrm{LvHg}$ or HvLg groups (Table 1, Fig. 1). This population also segregated the SSC4 QTL region that is marked by the WUR10000125 SNP [9], allowing a test of genotype effect in the same population. Animals with specific genotypes at this locus have been shown to have different infection response phenotypes in other PHGC studies [9]. For example, in the current dataset, we found that viremia levels were already significantly different between AA $(1461.9 \pm 175.6)$ and $\mathrm{AB}$ animals $(561.1 \pm 82.3)$ at $4 \mathrm{dpi}(p$-value $=0.005)$. A microarray analysis was then performed on RNA prepared from whole blood collected at 0,4 or $7 \mathrm{dpi}$ [22]. 
First, a linear model analysis was used to identify significant gene expression differences due to a PRRSv infection over time. Blood RNA levels at $4 \mathrm{dpi}$ versus $0 \mathrm{dpi}$, or $7 \mathrm{dpi}$ versus 0dpi were compared. Significant DE was found in 67 and 239 genes between 4dpi and 0dpi at an FDR of 0.05 and 0.10 , respectively (Table 2 ). The full list of DE genes is shown in Additional file 1: Table S1. For the 7dpi-0dpi dataset, 34 and 165 genes were found at an FDR of 0.05 and 0.10 , respectively (Table 2, Additional file 2: Table S2). GO term analyses were performed at an FDR of 0.10. They revealed that several immune response pathways were upregulated after infection (adaptive immune response, SH3 domain, regulation of $\mathrm{T}$ cell activation). However, enrichment scores for these GO terms were only marginally significant and therefore did not indicate a strongly upregulated pathway (Fig. 2). mRNA binding, translation initiation factor activity and mRNA processing activities were downregulated after infection.

Second, a linear model that contrasted different phenotypic extremes or WUR genotypes was performed to find differentially expressed genes. Expression differences were investigated between $\mathrm{Hg}$ and $\mathrm{Lg}$ animals within $\mathrm{Hv}$ or Lv groups on both $4 \mathrm{dpi}$ and 7dpi. A similar analysis was done to compare $\mathrm{Hv}$ and $\mathrm{Lv}$ animals within the $\mathrm{Hg}$ or $\mathrm{Lg}$ groups. Only the comparison between $\mathrm{LvHg}$ and LvLg animals on 7dpi showed sufficient DE genes (709) at an FDR of 0.10 to permit annotation enrichment analysis (Table 2, Fig. 2c). GO terms showed enrichment of the Toll-like signaling pathway in this

Table 2 Numbers of DE genes for the different comparisons

\begin{tabular}{|c|c|c|}
\hline & Comparison & $\begin{array}{l}\text { \# of DE genes } \\
(F D R=0.10)\end{array}$ \\
\hline \multirow[t]{4}{*}{ WG effect } & HvHg-HvLg contrast on 4dpi & 0 \\
\hline & HvHg-HvLg contrast on 7dpi & 0 \\
\hline & LvHg-LvLg contrast on 4dpi & 2 \\
\hline & LvHg-LvLg contrast on 7dpi & 709 \\
\hline \multirow[t]{4}{*}{ VL effect } & HvHg-LvHg contrast on 4dpi & 0 \\
\hline & HvHg-LvHg contrast on 7dpi & 0 \\
\hline & HvLg-LvLg contrast on 4 dpi & 2 \\
\hline & HvLg-LvLg contrast on 7dpi & 0 \\
\hline \multirow[t]{2}{*}{$\begin{array}{l}\text { WG and } V L \\
\text { interaction effect }\end{array}$} & $\begin{array}{l}(\mathrm{HvHg}-\mathrm{HvLg})-(\mathrm{LvHg}-\mathrm{Lv} L \mathrm{~g}) \\
\text { contrast on } 4 \mathrm{dpi}\end{array}$ & 1 \\
\hline & $\begin{array}{l}(\mathrm{HvHg}-\mathrm{HvLg})-(\mathrm{LvHg}-\mathrm{LvLg}) \\
\text { contrast on 7dpi }\end{array}$ & 0 \\
\hline \multirow[t]{2}{*}{ day effect } & 4dpi-0dpi contrast & 239 \\
\hline & 7dpi-0dpi contrast & 165 \\
\hline \multirow[t]{2}{*}{ genotype effect } & $\begin{array}{l}\text { genotype ( } A B \text { vs } A A) \\
\text { contrast on } 4 \mathrm{dpi}\end{array}$ & 0 \\
\hline & $\begin{array}{l}\text { genotype ( } A B \text { vs } A A) \\
\text { contrast on } 7 \mathrm{dpi}\end{array}$ & 0 \\
\hline
\end{tabular}

Comparisons were made for a WG, VL, day and genotype effect. The FDR was set at 0.10 contrast but, as above, this enrichment was only marginally significant. Interaction effects between the four quadrants did not identify many differentially expressed genes on either of the days, and a similar result was seen for the WUR genotype contrast (Table 2).

\section{Validation of the microarray results using RNAseq data}

To validate these microarray results, DE gene lists were compared with RNAseq data collected from blood samples of similarly infected animals. For the 4dpi-0dpi dataset, comparing the DE gene lists of genes with a q-value $\leq 0.05$ revealed that 11 of 31 genes present in both the microarray and the RNAseq experiment were significantly up- or downregulated (q-value $\leq 0.05$ for the microarray; q-value $\leq$ 0.10 for RNAseq) (Additional file 3: Table S3). The fold change of these genes measured by microarray and the fold change measured by RNAseq were significantly correlated overall (Pearson's correlation of $0.42 ; p$-value $=0.019)$. For the $7 \mathrm{dpi}$-0dpi dataset, the differential expression of only 1 of 15 genes was confirmed (Additional file 3: Table S3) and the correlation between the microarray and the RNAseq data was not significant (Pearson's correlation of $-0.30 ; p$-value $=0.28$ ). Since the microarray results could not be validated for the 7dpi-0dpi dataset, further network analyses were only performed on the 4dpi-0dpi dataset.

\section{WGCNA analysis on 4dpi-0dpi to identify co-expressed genes whose expression pattern was significantly correlated with the examined phenotypes/genotypes}

Because the one-gene-at-a-time analysis using a linear model failed to provide biological insight into the differences between pigs with extreme phenotypes after PRRSv infection, the focus then was on clusters of genes with a similar expression pattern rather than individual gene expression values. For this approach, the clustering tool WGCNA was used to analyze the 4dpi-0dpi dataset. To create the WGCNA modules, a soft threshold power of 3 was chosen, as suggested by the software as being the best threshold to create a scale free topology, while still giving a suitable amount of node connectivity. WGCNA created 18 modules based on the correlation of 4dpi-0dpi expression ratios across the 100 samples and they were assigned a color label. All uncorrelated genes were assigned to a grey module (Fig. 3). The correlations of all MEs with trial were examined and shown to be non-significant, as expected in trial-corrected data (data not shown). The correlations of MEs with WG and WG residuals or with VL and VL residuals were similar, so only correlations with WG and VL are further discussed. Four modules had MEs that were significantly correlated ( $p$-value $<0.1)$ with VL (midnightblue, blue, lightcyan and yellow), while three MEs were significantly 


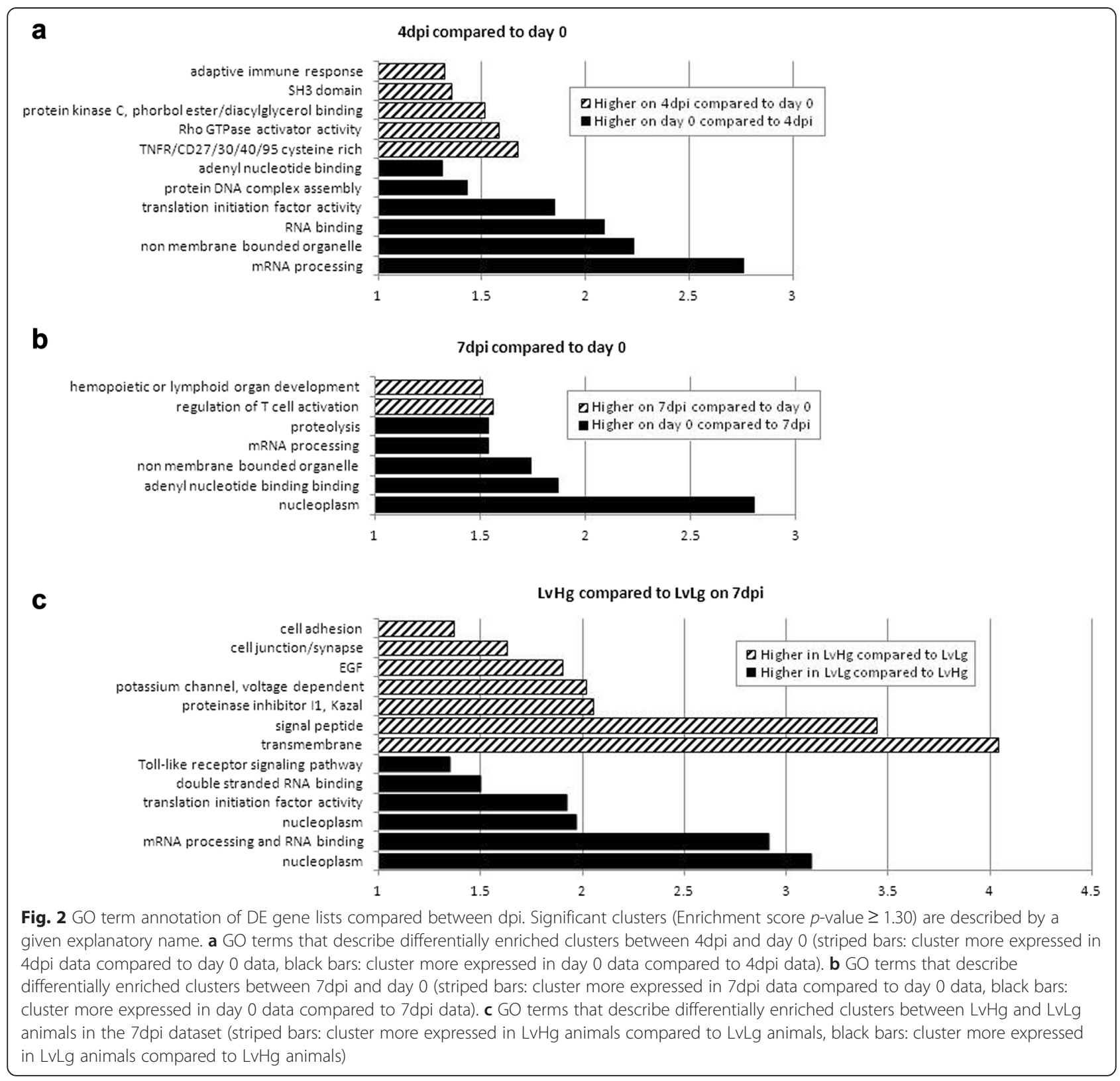

correlated with WG (red, lightgreen and cyan). Two of those three (red and cyan) were significantly correlated with WUR genotype as well. Furthermore, Additional file 4: Figure S1 shows the correlations between these modules and weight or serum viremia on specific days. Most of the modules that were significantly correlated with an overall WG or VL show particular time points that indicate a strong association with the gene expression patterns.

To evaluate the stability of these modules, networks were created with random subsets of the original samples (Additional file 5: Figure S2). Overall, the modules were consistent across all datasets created. BioLayout Express 3D (BE3D) was used to visualize the spatial relationship between the modules and their genes (Additional file 6: Figure S3) [42]. Genes whose individual expression profiles are correlated with at least one other gene at a Pearson correlation of $r=0.70$ are shown. The gene nodes were colored according to the module color assigned by WGCNA (Fig. 3).

\section{Annotation of the modules created by WGCNA}

To determine the biological relevance of these correlations, GO enrichment was examined in gene lists of modules whose ME was significantly correlated with traits of interest. Additional file 7: Table S4 gives an overview of the enriched GO terms of these module gene lists, the number of genes in each, the number of 


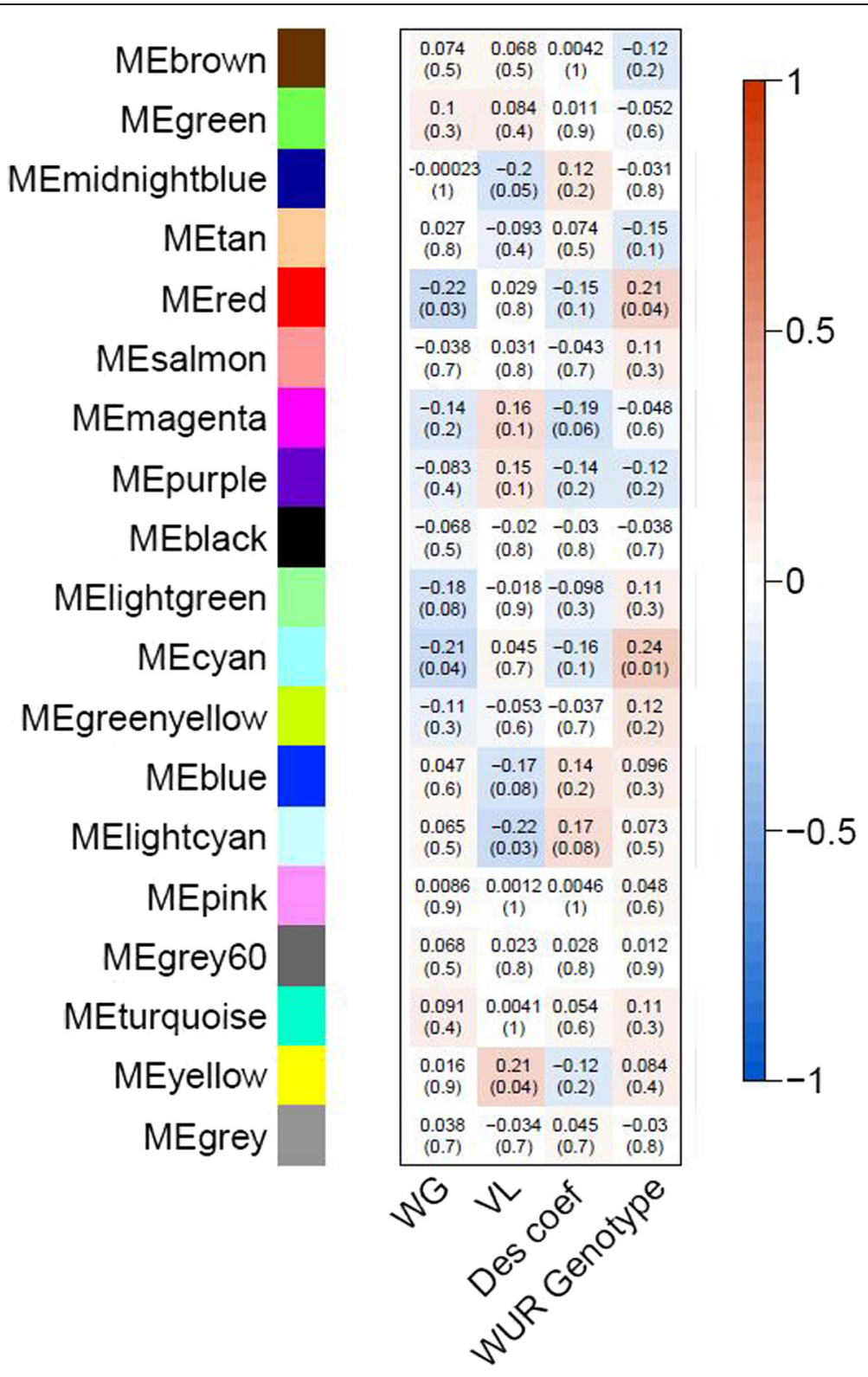

Fig. 3 WGCNA modules and the MEs' correlations with the traits of interest for 4dpi-Odpi comparison. Horizontally, MEs are named by the module colors, arbitrarily assigned by WGCNA. Vertically, phenotypes of interest are listed (WG, VL, Des coef and WUR genotype). Numbers given are the correlation coefficients between the respective ME and the trait of interest, with nominal $p$-value between brackets. The more intensely blue a box is colored, the more negatively its correlation is with that trait, the more intensely red, the more positive the correlation with that trait

available human Ensembl gene IDs for those genes and how many of them were recognized by DAVID. An explanatory name was chosen to describe the cluster based on the GO terms enriched.

When performing a WGCNA analysis on the 4dpiOdpi dataset, a total of 7 modules were significantly correlated with VL or WG ( $p$-value $<0.1)$. GO enrichment analysis for modules significantly correlated with VL was not very informative. The blue module ( $p$-value $=0.08)$, which contained over 2300 genes, was enriched in annotation for very broad terms such as transmembrane, amino acid transmembrane transporter and ion transport. Analysis of other significant modules for VL (midnightblue: $p$-value $=0.05$, lightcyan: $p$-value $=0.03$ and yellow: $p$-value $=0.04$ ) also did not reveal explicit pathways. For WG, the modules that were significantly correlated with the trait were all negatively correlated (lightgreen: $p$-value $=0.08$, cyan: $p$-value $=0.0$ and red: 
$p$-value $=0.03)$, indicating that the overall expression of these genes was higher in the animals having a lower WG. Two modules whose ME was significantly correlated with WUR genotype (cyan: $p$-value $=0.01$; red: $p$-value $=0.04)$, had a ME that was also correlated with WG, in a direction that indicates that $A B$ animals have a desirable PRRS response phenotype (higher weight gain post-infection) when compared to AA animals, as was discovered by Boddicker et al. [9]. The light green module, which contained only 49 genes, did not show any significant enrichment. The cyan module contained 127 genes and was annotated as a module important for RNA processing, chromosomal organization and DNA replication. The red module, containing 506 genes, was most immunologically relevant, with GO terms such as innate immune response, Toll-like receptor signaling pathway and complement activation.

To see if a significant correlation of the modules was primarily due to an up- or downregulation of expression of genes in that cluster, or whether it could be partly explained by specific cell types, a cell type enrichment analysis was performed on both datasets using the web-based tool CTEN (Fig. 4). Eleven modules showed enrichment for gene expression patterns observed for specific cell types $(p \leq 0.01)$. Within these, the midnightblue, red and cyan modules were significant for a trait of interest, as mentioned earlier.
PCIT analysis on 4dpi-0dpi to find regulatory differences between phenotypic groups and between animals having a different WUR genotype

A PCIT analysis explores differences in connectivity strength between gene expression patterns in two contrasting groups of animals, as measured by connections drawn between genes in a correlation network. Genes shown to have significant differences in connections between groups with a distinct set of genes are called hub genes and could identify a difference in gene regulation between these groups. In this study, the phenotypic contrasts provided to PCIT were $\mathrm{Hg}$ versus $\mathrm{Lg}, \mathrm{Hv}$ versus Lv, High Des coef $(>0.5)$ versus Low Des coef $(<0.5)$, and $A A$ versus $A B$ WUR genotype. Additional file 8: Table S5 shows for each contrast the top 10 differentially connected hub genes, their annotation and the difference in number of correlates between the two contrasts. For these PCIT analyses, Information Theory was used to determine the significance of correlation in these PCIT analyses. This approach considers the total number of correlations calculated for the entire dataset and only the partial correlation values that achieve the significance threshold for the entire dataset are retained to define the connectivity between a target and hub gene. To explore the effect of these differences, all correlates of the 10 extreme DH genes were combined and the resulting gene list examined for GO term enrichment. In

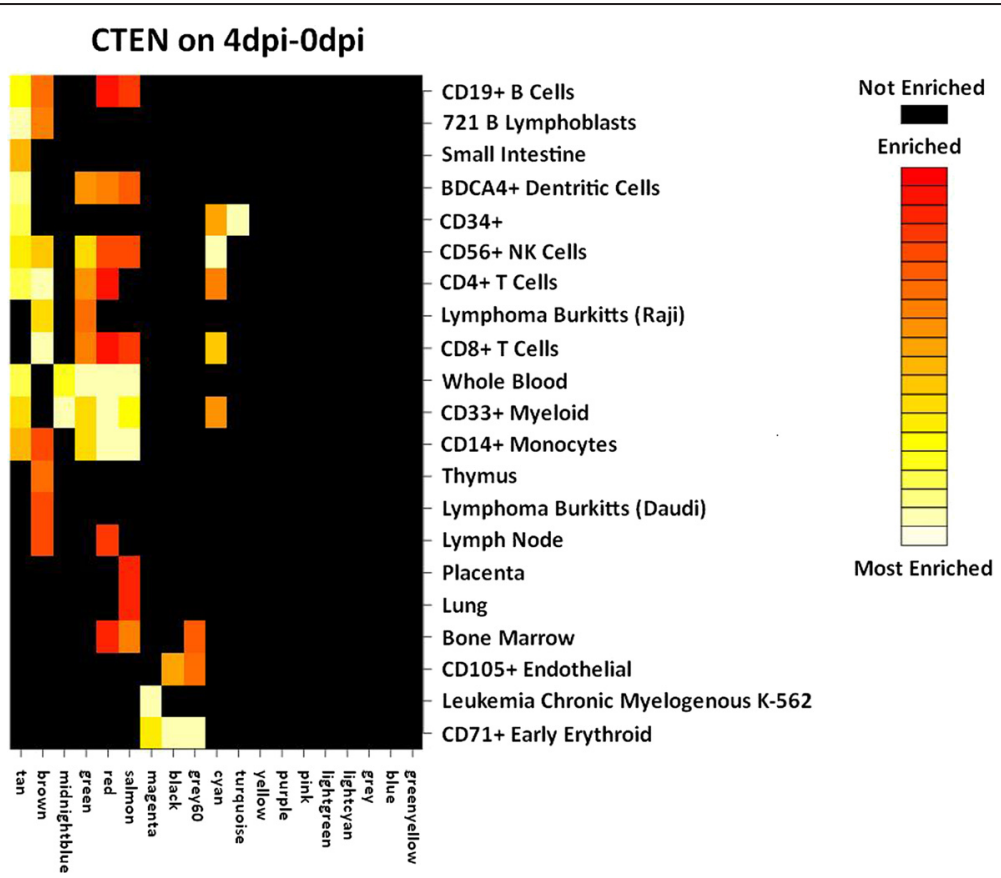

Fig. 4 Cell Type Enrichment (CTEN) analyses on the 4dpi-Odpi dataset. The WGCNA clusters are noted along the abscissa. Black squares represent no enrichment in cell type. From red to yellow to white are all significant enriched cell-type specific genes within each module gene list (enrichment score $\geq 2.0$ ), going from enriched to most enriched 
Fig. 5a, significant GO annotation terms are shown for the correlates that were only present in the Lv group or more strongly connected to at least one of the top 10 extreme hub genes in the Lv group when compared to the $\mathrm{Hv}$ group. The height of the bars is the average $\log _{2}$ fold change between 4dpi and 0dpi of all correlates annotated with the respective $\mathrm{GO}$ term. Figure $5 \mathrm{~b}$ shows the enriched annotation of correlates of the hub genes in the Hv group. Several immune-related pathways were significantly over-represented in the Lv group compared to the $\mathrm{Hv}$ group. Similar results for GO terms of correlates tightly connected to the $\mathrm{Lg}$ and $\mathrm{Hg}$ group are shown in Fig. $5 \mathrm{c}$ and $\mathrm{d}$, with more enrichment of immune-related pathways in the Lg group compared to the $\mathrm{Hg}$ group. Animals with a low Des coef $(n=34)$ were contrasted with the animals with a high Des coef $(n=38)$ in Fig. 6a and $b$. The GO terms enriched for the correlates of the top 10 hub genes in $A A$ and $A B$ animals is shown in Fig. $6 \mathrm{c}$ and d. For these last two contrasts, GO terms did not reveal major correlation network differences between more and less favorable animals.

\section{Discussion}

In this study, systemic differences among 100 animals that exhibited diverse responses to PRRSv infection were explored using microarray analysis of whole genome blood expression levels. Although a substantial number of animals that represented each of the four extreme phenotypic groups were evaluated in a linear model based approach, few differentially expressed genes were found. The linear model declared a relatively small number of genes upregulated at $4 \mathrm{dpi}$ and $7 \mathrm{dpi}$ when compared to 0dpi, while DE genes were found in a single phenotypic group comparison, and this only when looking at the 7dpi expression profiles. GO annotation enrichment analyses of these lists did illustrate some upregulated immune responses after infection for both $4 \mathrm{dpi}$ and $7 \mathrm{dpi}$, and in the $\mathrm{Lg}$ group compared to the $\mathrm{Hg}$

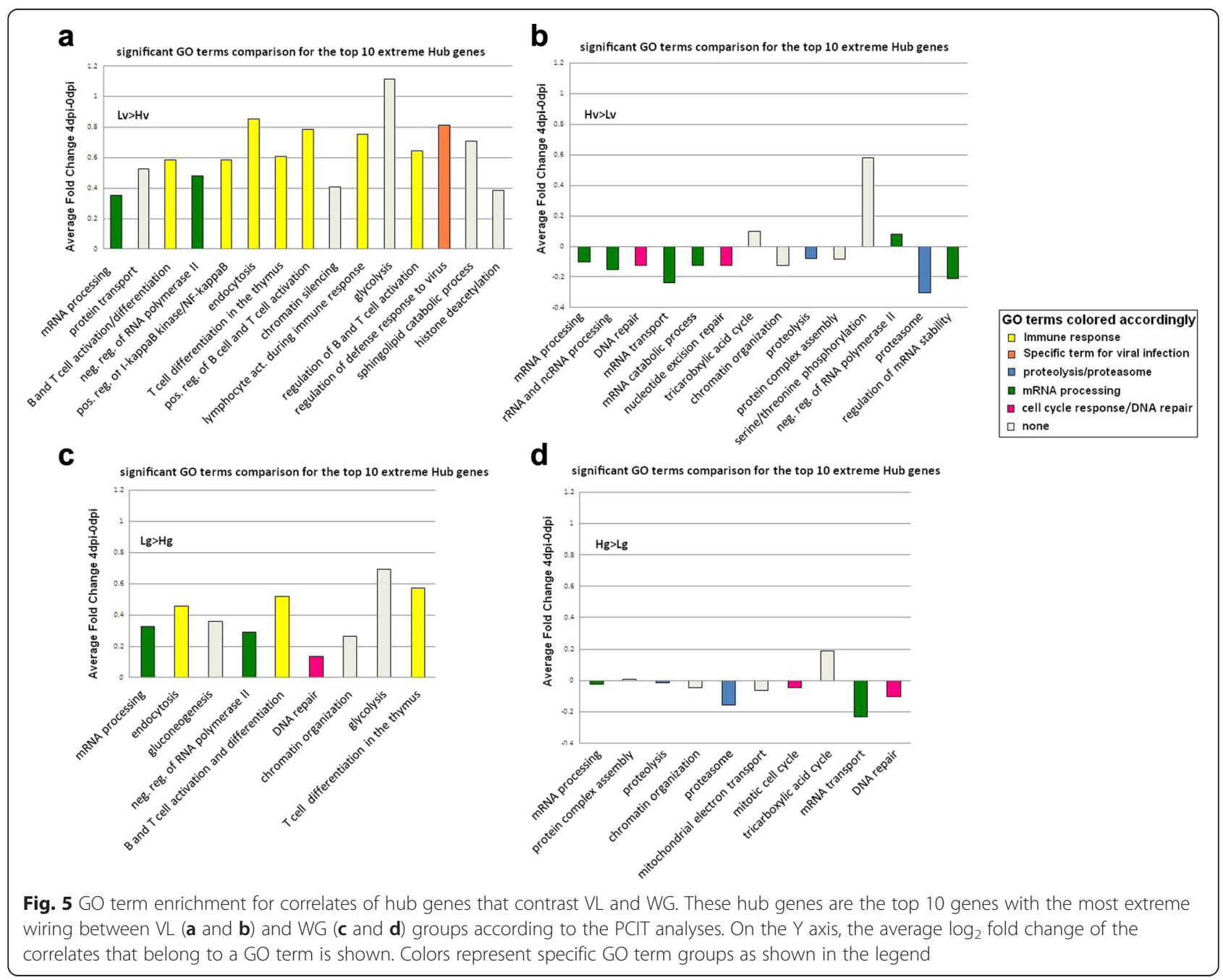



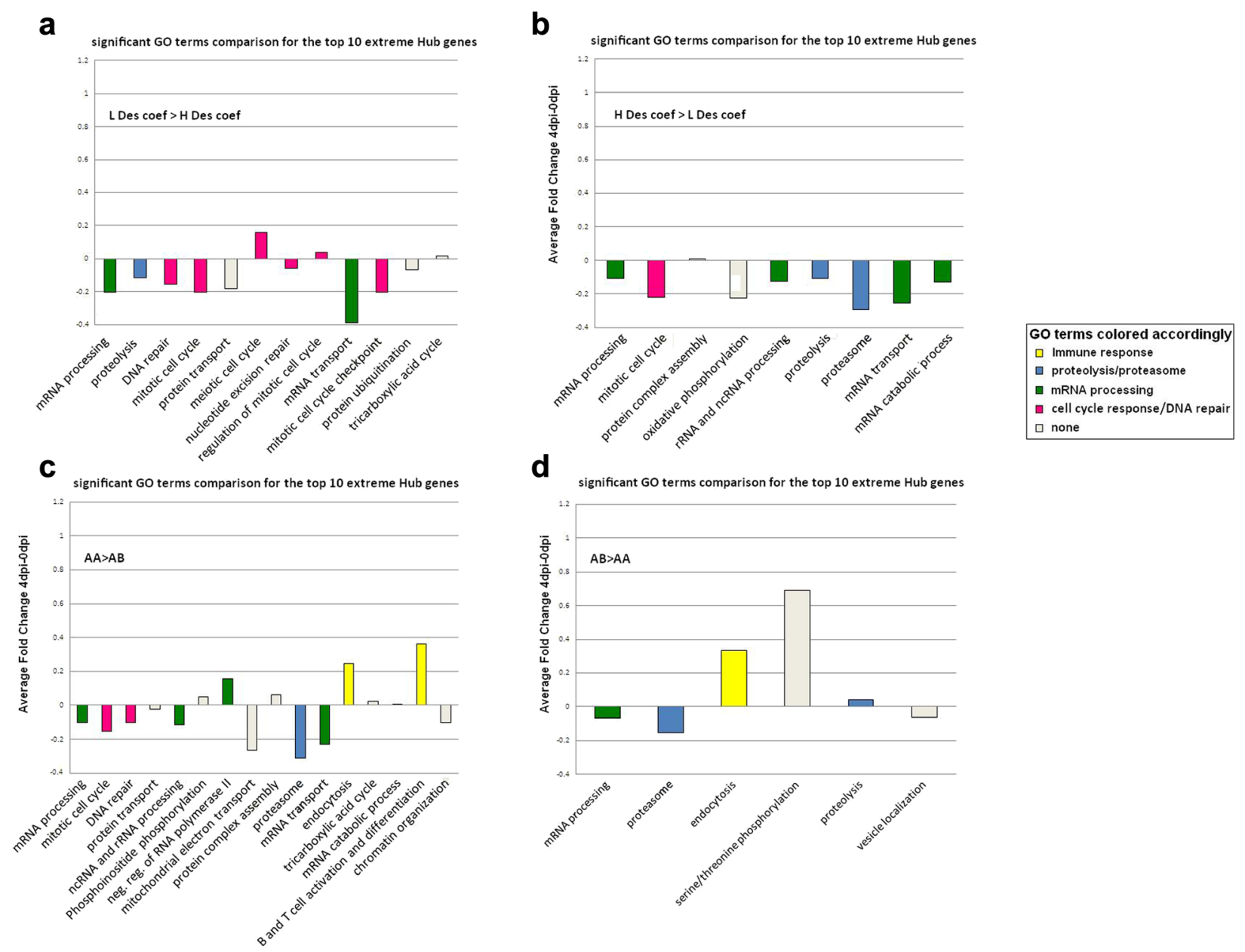

Fig. $6 \mathrm{GO}$ term enrichment for correlates of hub genes that contrast Des coef and WUR genotype. These hub genes are the top 10 genes with the most extreme wiring between Des coef ( $\mathbf{a}$ and $\mathbf{b}$ ) and WUR genotype ( $\mathbf{c}$ and $\mathbf{d}$ ) groups according to the PCIT analyses. On the $Y$ axis, the average fold change of the correlates belonging to a GO term is shown. Colors represent specific GO term groups as shown in the legend

group at 7 dpi. Nevertheless, this analysis found few terms were enriched within these gene lists, and therefore a limited amount of biological insight could be gleaned from these analyses.

In addition, after trying to validate the gene expression pattern differences measured by the microarray between $4 \mathrm{dpi}$ or $7 \mathrm{dpi}$ and $0 \mathrm{dpi}$, it became clear that the $7 \mathrm{dpi}-0 \mathrm{dpi}$ dataset was difficult to validate, as was also seen earlier by Arceo et al. [22]. To validate these data, RNAseq data from blood taken from an independent set of animals was used. Although both RNAseq and microarray analyses measure the transcriptome, studies report different genes as DE using one method or the other, even when using the exact same samples [43-45]. In addition, the difficult validation of the $7 \mathrm{dpi}$ dataset may also be due to the fact that different groups of animals were analyzed by the two techniques, and at 7dpi, the virus is still replicating in some animals while in others the adaptive immune response might already be ramping up. Thus, significant variation is likely present in the response to PRRSv observed at $7 \mathrm{dpi}$ across experimental groups. For these reasons, we decided to only examine the $4 \mathrm{dpi}-0 \mathrm{dpi}$ dataset in further analyses.

The main goal of this study was not to investigate differences due to infection, but to explore whether there were differences between the phenotypic groups that diverged after infection. Therefore, other more sensitive analyses were performed. These more sensitive coexpression analyses emphasize differences in gene network up- or downregulation rather than individual gene expression differences [46].

The red module is related to inflammation and its average expression is negatively correlated with weight gain after a PRRSv infection

The red module, with 506 genes, was enriched for several immune response terms and could therefore contain genes of great importance in a PRRSv infection. 
An overview of the genes in the red module at 4dpi-0dpi is shown in Additional file 9: Table S6. The CTEN analysis revealed that the red module genes were enriched in genes specifically expressed in CD33+ myeloid cells and CD14+ monocytes. This could indicate that the expression patterns of the genes in this module were not solely due to transcriptional changes but possibly also due to a difference in monocyte recruitment into the blood. It has been described that PRRSv infection causes an increase in CD14+ expression throughout the early stage of infection, due to a rise in CD14+ monocytes that differentiate to macrophages and migrate to bronchoalveolar spaces [47]. The red module contained several anti-apoptosis members of the BCL2 family (BCL2A1, BCL2L14, BCL2L15, MCL1) earlier reported to be upregulated after PRRSv infection [14, 15]. Antiapoptosis is often seen in the early stage of a PRRSv infection: this is beneficial for the virus since anti-apoptosis expands time for viral replication in macrophages [48, 49]. Only later in the infection do PRRSv infected macrophages die by apoptosis [48]. A large portion of genes present in the red module encodes pathogen-recognition receptors (PRRs) such as Toll-like receptors (TLR2, TLR4) and the CD14 co-receptor for TLR4 (CD14), C-type lectin receptors (CLEC2B, CLEC2D, CLEC4E, CLEC7A) and RIG-1-like receptors (DDX58 or RIG1, MDA5 or IFIH1). PRRs play a main role in triggering inflammatory responses by activating and inducing release of cytokines, chemokines and type I interferons (IFNs) [50]. Additionally, several members of this module are known as interferon-stimulated genes (OAS1, RNASEL, MB21D1, BST2, IFIT3, ISG20, RSAD2, DDX60, USP18) [51]. Many of these genes where also found in the type I interferon/ cytokine mediated immune response cluster found by the Immune Response Annotation Group [52]. However, genes, seen to have a specific PRRS response expression pattern, elicited by macrophages or lymph nodes, were not clustered in this red module, nor in any other cluster. Type I interferons induce an innate antiviral response, and PRRSv infection has been observed to dampen or delay the type I interferon transcriptional response [19, 53, 54]. Ait-Ali et al. [19] described a difference in PRRS susceptibility between macrophages isolated from Landrace versus Piétrain pigs. The main difference was the rate with which type I interferon transcriptional changes occurred: they suggested this as the underlying reason for breed differences in PRRS susceptibility [19]. Souza et al. [55] reported that IFN $\alpha$ levels in serum increased rapidly after a PRRS infection. However, after 4dpi, the best animals (LvHg group) had the fastest return to basal levels; as early as $7 \mathrm{dpi}$, the IFNa levels were significantly lower in this group compared to other phenotype quadrants [55]. In our study, the red module, that contained several interferon-stimulated genes, was found to be negatively correlated with WG and positively with WUR genotype, which meant that animals with a lower weight at 42dpi or animals with the unfavorable genotype for WUR expressed higher levels of these genes at $4 \mathrm{dpi}$. Because a significant difference in viremia was noticeable between the two WUR genotype groups already at 4dpi in these pigs, we propose that the effect of genotype on phenotype is already present by this time. Since the red module was positively correlated with viremia at $4 \mathrm{dpi}$, our hypothesis is that the resistant $A B$ animals control virus replication through red module interferon-stimulated genes prior to 4dpi. This earlier, and possibly more effective response, is supported by IFN $\alpha$ data on similarly challenged PHGC cohorts, which shows that $\mathrm{LvHg}$ animals more quickly bring down their IFN $\alpha$ serum levels. This drop is significantly faster and to lower levels for these $\mathrm{LvHg}$ animals compared to the other groups, indicating a faster initiation of the resolution of the anti-viral response [55]. Hulst et al. [56] showed that type I interferon expression in the blood can differ between susceptible and more resistant pigs to a classical swine fever infection. In their study, no significant pathways involved in the induction of the antiviral IFN type I response were upregulated in chronically diseased pigs at $4 \mathrm{dpi}$ or $8 \mathrm{dpi}$, while in pigs that recovered rapidly, these pathways were already significantly upregulated at 4dpi until recovery at 10-12dpi [56], which supports our theory that better adapted animals induce the interferon type I response faster. We further speculate that susceptible AA animals demand more energy by keeping their immune system active longer (higher expression in blood of red module genes at $4 \mathrm{dpi}$ ), which could manifest in poor growth in the long run [57].

Besides interferon-stimulated genes, other inflammatory response genes were found in the red module. Miller et al. [13] recently described the infection response in tracheobronchial lymph nodes to two PRRSv strains, a Chinese highly pathogenic rJXwn06 strain and the US VR-2332 strain. They found that the RNAs for serum amyloid A2 acute phase proteins, S100A8, S100A9 and S100A12, were amongst the top upregulated genes in animals inoculated with these strains compared to sham inoculated pigs [13]. S100A8 and s100A12 are found in the red module. Another gene in this module was TREM1, known to trigger release of pro-inflammatory chemokines and cytokines, and found by Miller et al. [13] and Badaoui et al. [12] to be highly induced after PRRSv infection. Furthermore, NLRP3, CASP1, IL1B and IL18 were also found in the red module. The inflammasome gene NLRP3 is known to activate $C A S P 1$, which leads to the activation of $I L 1 B$ and IL18 [58]; all have been previously shown to be upregulated after PRRSv infection [59]. These inflammatory responses are of interest with regard to the different WUR genotypes, since the region near the WUR SNP in the SSC4 region contains several immune-related genes such 
as members of the Leucine Rich Repeat Containing protein 8 (LRRC8) family [60] and genes belonging to the Guanylate Binding Protein (GBP) family [61]. Recently, a putative causal mutation for the SSC4 PRRS host response QTL associated with WUR was identified in GBP5 [35]. Since GBP5 directly regulates NLRP3 inflammasome assembly, it is interesting that the red module includes NLRP3 and several of the inflammatory genes that it regulates. Finally, Wysocki et al. [21] have described the importance of the complement cascade during PRRSv response, and the red module contains genes encoding several complement regulatory proteins such as C4BPA, the decay-accelerating factor (DAF) CD55, the membrane cofactor of proteolysis (MCP) CD46 and the C1 inhibitor SERPING1, all inhibitors of complement activation [62]. This points to a difference in complement regulation in the slower growing animals, or those that have the unfavorable WUR genotype.

\section{PCIT analysis clearly indicates immune network differences between phenotypic divergent animals early after PRRSv infection}

In a next step, PCIT was used to explore the regulatory networks that changed between animals with different phenotypes or genotypes at $4 \mathrm{dpi}$. The largest difference in GO terms enriched in the group of correlates could be seen in those for lymphocyte activation, and this effect was strongest when the viral load phenotypes were contrasted. In other words, animals that belonged to the Lv group had top hub genes with tighter connections to genes in the immune activation pathways than did the $\mathrm{Hv}$ group at $4 \mathrm{dpi}$. This difference was also noticeable when looking at the GO term enrichment of the correlates of the 10 extreme hub genes in the $\mathrm{Hg}$ and Lg groups, where animals showed tighter connections to immune genes in the Lg group. As discussed previously, this result can be explained by the allocation of energy to immune response activation rather than growth. Thus, the Lg animals may partition more energy to immune response and have less energy to put towards weight gain. The difference in connection to the immune genes disappeared when looking at the Low Des coef animals, that had high viremia levels and had a reduced growth, in comparison to the better High Des coef animals. There are only small differences between the unfavorable AA animals and the favorable $\mathrm{AB}$ animals, with the most apparent difference in enriched $B$ cell and $\mathrm{T}$ cell differentiation GO terms in the AA animals. The top different hub genes in the networks between the most and least desirable phenotype ( $\mathrm{LvHg}$ versus $\mathrm{HvLg}$, respectively) or genotype ( $A B$ versus $A A$, respectively) did not differ greatly in their connection to immune genes.

\section{Do WGCNA and PCIT methods agree with one another?}

As seen earlier, the red module from the WGCNA analysis, annotated as a highly immune gene rich module, was negatively correlated with weight gain and more highly expressed in the AA animals. This agrees with the PCIT results, where immune response GO terms were more enriched in the correlates of the $\mathrm{Lg}$ over the $\mathrm{Hg}$ group and, to a lower extent, the $A A$ versus $A B$ animals. However, it is not predominantly the genes in the red module that were more tightly correlated in the PCIT analyses. Combining results across these analyses, it is clear that the immune response pathways regulated at 4 dpi play a substantial role when defining the pig's longterm phenotypic response to a PRRSv infection, as affirmed by Boddicker et al. [9].

Furthermore, in Fig. 3 it can be seen that modules of co-expressed genes are correlated with weight gain, viral load or neither of these two, but never with weight gain and viral load simultaneously. A significant correlation of the Des coef with the ME in the magenta and lightcyan module was driven by only one of the two phenotypes. The PCIT results indicated that in this 4dpi-0dpi dataset it is more difficult to find co-expressed gene clusters correlated with both phenotypes in a desired direction. From the earliest PHGC trials it was clear that VL and WG were only poorly correlated; however, the SSC4 allele perfectly negatively correlated WG with VL $[9,10]$. Other genomic influences would be predicted to influence just one not both traits. The greatest difference in GO term enrichment of the correlates of top 10 hub genes for both VL and WG are both immune response terms: they are more strongly connected with the desired phenotype for VL, but at the same time, also with the undesired phenotype for WG.

\section{Conclusion}

In this study, it became clear that a gene-by-gene linear modeling analysis was not sufficiently sensitive to find expression differences in the blood early after infection between animals with a different weight gain or viral load after a PRRSv infection or between animals with the favorable and unfavorable WUR genotype. However, the network-level approaches WGCNA and PCIT successfully found co-expressed immune response genes at $4 \mathrm{dpi}$ when compared to 0dpi to be correlated as a group with weight gain, viral load or WUR genotype. Thus these genes can be useful targets in future efforts to select for disease-resistant pigs.

\section{Availability of supporting data}

All microarray experimental data are MIAME compliant and have been deposited in Gene Expression Omnibus (GEO) with the accession number: GSE69515 (http:// www.ncbi.nlm.nih.gov/geo/query/acc.cgi?acc=GSE69515). 


\section{Additional files}

Additional file 1: Table S1. List of DE genes (FDR $\leq 0.10$ ) between 4dpi and Odpi. The Oligo ID is a unique microarray probe name of the transcript. HGNC, extra annotation and HGNC extra annotation are different annotations. For all genes, the $\log _{2}$ fold change is shown, as well as the nominal $p$-value and q-value for that fold change.

Additional file 2: Table S2. List of DE genes (FDR $\leq 0.10)$ between 7dpi and Odpi. The Oligo ID is a unique microarray probe name of the transcript. HGNC, extra annotation and HGNC extra annotation are different annotations. For all genes, the $\log _{2}$ fold change is shown, as well as the nominal $p$-value and q-value for that fold change.

Additional file 3: Table S3. List of genes selected for validation of the microarray results with RNAseq data. 31 and 15 genes were differentially expressed in the micro-array and found in the RNAseq experiment, and these were used to validate the DE between 4dpi and Odpi and between $7 \mathrm{dpi}$ and $0 \mathrm{dpi}$, respectively. For every gene, the probe is indicated by the unique Oligo ID, and the HGNC of each gene is shown. For both the microarray and RNAseq data, the DE is specified by the $\log _{2}$ fold change on the respective day, as well as the nominal $p$-value and q-value for that fold change. The last three columns show the comparison between the two datasets: when the gene fold change in both datasets goes in the same direction, it is indicated with a " + ", if this change in the RNAseq is significant, it is indicated with a " + ", and if both direction and significance are similar for both datasets, the validation is confirmed and indicated with a " + ".

Additional file 4: Figure S1. Modules and their correlations with weight and blood viremia on specific days. Weight was measured at 0, 7, $14,19 / 21,28,35$ and 40/42 dpi, viremia was examined at 4, 7, 11, 14 and 19/21 dpi.

Additional file 5: Figure S2. Stability analysis for the 4dpi-0dpi dataset. From the $4 \mathrm{dpi}-0 \mathrm{dpi}$ dataset 50 random samplings were performed and modules were created to evaluate the stability of the original full dataset.

Additional file 6: Figure S3. WGCNA clusters shown with BioLayout Express 3D. Nodes are colored according to the module color assigned by WGCNA. All transcripts with a Pearson correlation of $R^{2}>0.70$ are kept.

Additional file 7: Table S4. Annotation of significant WGCNA modules in the 4dpi-Odpi comparison using DAVID. GO term descriptions are shown for those modules in the $4 \mathrm{dpi}-0 \mathrm{dpi}$ dataset that were significantly correlated ( $p$-value $<0.10)$ with at least one of the phenotypes examined. Total number of transcripts in a module is given, as well as how many of them could be linked to a human Ensembl Gene ID and of those, how many were recognized by DAVID. An enrichment score (Enrichment score $p$-value $\geq 1.30$ ) for each annotation is given as well. In orange is the 4dpi-0dpi red module, discussed in more detail in the discussion.

Additional file 8: Table S5. PCIT results for the 4dpi-0dpi comparison for the four different contrasts. Contrasts made were done according to genotype, WG, VL and Des coef. The top 10 hub genes for every comparison are shown. The Oligo ID column shows a unique microarray probe name of the transcript. The HGNC column shows the annotated gene name, when available. The PCIT score represents the difference in amount of correlates. The group with the most correlates is named in the first column. Module color is the color for the hub gene given by WGCNA in the 4dpi-0dpi dataset.

Additional file 9: Table S6. Pigoligoarray probes present in the red module of the $4 \mathrm{dpi}-0 \mathrm{dpi}$ dataset. The Oligo ID column shows a unique microarray probe name of the transcript. HGNC, extra annotation and HGNC extra annotation are different annotations. The Gene Significance (GS) and its $p$-value for each gene in relation to the trait WG, VL and WUR genotype in the depicted module is shown. The higher the absolute value of this GS, the more biologically significant the gene is for the module with regard to the trait. In orange are those genes discussed in more detail.

\section{Abbreviations}

BE3D: BioLayout Express 3D; CTEN: Cell type enrichment; DAVID: Database for Annotation, Visualization and Integrated Discovery; DE: Differential expression; Des coef: Desirability Coefficient; DH: Differential hubbing; dpi: Days post infection; FDR: False Discovery Rate; GEO: Gene Expression Omnibus; GO: Gene Ontology; H: High responders; HvHg: High VL with maximal WG.; HVLg: High VL with reduced WG; IACUC: Institutional Animal Care and Use Committee; L: Low responders; LIMMA: Linear Models for Microarray Data; LVHg: Low VL with maximal WG; LVLg: Low VL with reduced WG; ME: Module eigengene; NCBI: National Center for Biotechnology Information; NHS: N-hydroxysuccinate; PCIT: Partial Correlation and Information Theory; PHGC: PRRS Host Genetics Consortium; PRRSv: Porcine Reproductive and Respiratory Syndrome virus; QTL: Quantitative Trait Locus; SD: standard deviation; SNP: Single Nucleotide Polymorphism; SSC4: SUS scrofa chromosome 4; TCID50: 50 \% Tissue Culture Infective Dose; TMM: Trimmed Mean of M-values; VL: Viral load; WG: Weight gain; WGCNA: Weighted Gene Co-expression Network Analysis; WUR: WUR10000125.

\section{Competing interests}

The authors declare that they have no competing interests.

\section{Authors' contributions}

MS and JPS wrote the paper. CKT, CWE, JKL, IC, JEK, JMR and JCMD edited the paper. CKT, CWE, JKL, JPS conceived the study and developed the microarray experimental design. RRRR organized the animal trials and the collection of samples and phenotypes. IC performed RNA isolation. CWE and NER performed the microarray analyses. JPS conducted the statistical analysis for the microarray experiment. JEK, EF-W and JMR conducted the RNAseq bioinformatics. CJE and JCMD performed the RNAseq statistical analysis. MS executed WGCNA and CTEN analyses. JEK ran the PCIT and DH analyses. All co-authors have reviewed the manuscript.

\section{Acknowledgments}

This work was supported by USDA ARS funding and USDA NIFA grant \#2010-65205-20433. The PRRS Host Genetics Consortium (PHGC) samples were supported through grants \#07-233, \#09-244 and \#10-033 from the U.S. National Pork Board. The authors would like to thank Dr. Zhiliang Hu for the annotation of the Pigoligoarray.

\section{Author details}

'Department of Animal Science, lowa State University, Ames, IA, USA. ${ }^{2}$ Department of Animal Science, Michigan State University, East Lansing, MI, USA. ${ }^{3}$ Department of Fisheries and Wildlife, Michigan State University, East Lansing, MI, USA. ${ }^{4}$ APDL, BARC, ARS, USDA, Beltsville, MD, USA. ${ }^{5}$ Department of Statistics, lowa State University, Ames, IA, USA. ${ }^{6}$ Department of Diagnostic Medicine/Pathobiology, College of Veterinary Medicine, Kansas State University, Manhattan, KS, USA.

Received: 4 February 2015 Accepted: 30 June 2015

Published online: 10 July 2015

\section{References}

1. Holtkamp DJ, Kliebenstein JB, Neumann EJ, Zimmerman JJ, Rotto HF, Yoder TK, et al. Assessment of the economic impact of porcine reproductive and respiratory syndrome virus on United States pork producers. I Swine Health Prod. 2013;21(2):72-84.

2. Neumann EJ, Kliebenstein JB, Johnson CD, Mabry JW, Bush EJ, Seitzinger $\mathrm{AH}$, et al. Assessment of the economic impact of porcine reproductive and respiratory syndrome on swine production in the United States. J Am Vet Med Assoc. 2005;227(3):385-92.

3. Done SH, Paton DJ, White ME. Porcine reproductive and respiratory syndrome (PRRS): a review, with emphasis on pathological, virological and diagnostic aspects. Br Vet J. 1996;152(2):153-74.

4. Meulenberg JJ, Hulst MM, de Meijer EJ, Moonen PL, den Besten A, de Kluyver EP, et al. Lelystad virus, the causative agent of porcine epidemic abortion and respiratory syndrome (PEARS), is related to LDV and EAV. Virology. 1993;192(1):62-72.

5. Lunney JK, Benfield DA, Rowland RR. Porcine reproductive and respiratory syndrome virus: an update on an emerging and re-emerging viral disease of swine. Virus Res. 2010;154(1-2):1-6.

6. Kimman TG, Cornelissen LA, Moormann RJ, Rebel JM, Stockhofe-Zurwieden N. Challenges for porcine reproductive and respiratory syndrome virus (PRRSV) vaccinology. Vaccine. 2009;27(28):3704-18. 
7. Otake S, Dee S, Corzo C, Oliveira S, Deen J. Long-distance airborne transport of infectious PRRSV and Mycoplasma hyopneumoniae from a swine population infected with multiple viral variants. Vet Microbiol. 2010;145(3-4):198-208.

8. Lunney JK, Steibel JP, Reecy JM, Fritz E, Rothschild MF, Kerrigan M, et al. Probing genetic control of swine responses to PRRSV infection: current progress of the PRRS host genetics consortium. BMC Proc. 2011:5 Suppl 4:S30.

9. Boddicker N, Waide EH, Rowland RR, Lunney JK, Garrick DJ, Reecy JM, et al. Evidence for a major QTL associated with host response to porcine reproductive and respiratory syndrome virus challenge. J Anim Sci. 2012;90(6):1733-46.

10. Boddicker NJ, Garrick DJ, Rowland RR, Lunney JK, Reecy JM, Dekkers JC. Validation and further characterization of a major quantitative trait locus associated with host response to experimental infection with porcine reproductive and respiratory syndrome virus. Anim Genet. 2013;45(1):48-58.

11. Boddicker NJ, Bjorkquist A, Rowland RR, Lunney JK, Reecy JM, Dekkers JC. Genome-wide association and genomic prediction for host response to porcine reproductive and respiratory syndrome virus infection. Genet Sel Evol. 2014;46:18.

12. Badaoui B, Rutigliano T, Anselmo A, Vanhee $M$, Nauwynck $H$, Giuffra $E$, et al. RNA-sequence analysis of primary alveolar macrophages after in vitro infection with porcine reproductive and respiratory syndrome virus strains of differing virulence. PLoS One. 2014;9(3):e91918.

13. Miller LC, Fleming D, Arbogast A, Bayles DO, Guo B, Lager KM, et al. Analysis of the swine tracheobronchial lymph node transcriptomic response to infection with a Chinese highly pathogenic strain of porcine reproductive and respiratory syndrome virus. BMC Vet Res. 2012;8:208.

14. Xiao S, Jia J, Mo D, Wang Q, Qin L, He Z, et al. Understanding PRRSV infection in porcine lung based on genome-wide transcriptome response identified by deep sequencing. PLoS One. 2010;5(6):e11377.

15. Xiao S, Mo D, Wang Q, Jia J, Qin L, Yu X, et al. Aberrant host immune response induced by highly virulent PRRSV identified by digital gene expression tag profiling. BMC Genomics. 2010;11:544.

16. Zhou $P$, Zhai S, Zhou X, Lin P, Jiang T, Hu X, et al. Molecular characterization of transcriptome-wide interactions between highly pathogenic porcine reproductive and respiratory syndrome virus and porcine alveolar macrophages in vivo. Int J Biol Sci. 2011;7(7):947-59.

17. Genini S, Delputte PL, Malinverni R, Cecere M, Stella A, Nauwynck HJ, et al. Genome-wide transcriptional response of primary alveolar macrophages following infection with porcine reproductive and respiratory syndrome virus. J Gen Virol. 2008:89(Pt 10):2550-64

18. Xing J, Xing F, Zhang C, Zhang Y, Wang N, Li Y, et al. Genome-wide gene expression profiles in lung tissues of pig breeds differing in resistance to porcine reproductive and respiratory syndrome virus. PLoS One. 2014;9(1):e86101.

19. Ait-Ali T, Wilson AD, Carre W, Westcott DG, Frossard JP, Mellencamp MA et al. Host inhibits replication of European porcine reproductive and respiratory syndrome virus in macrophages by altering differential regulation of type-l interferon transcriptional response. Immunogenetics. 2011;63(7):437-48.

20. Bates JS, Petry DB, Eudy J, Bough L, Johnson RK. Differential expression in lung and bronchial lymph node of pigs with high and low responses to infection with porcine reproductive and respiratory syndrome virus. J Anim Sci. 2008;86(12):3279-89.

21. Wysocki M, Chen H, Steibel JP, Kuhar D, Petry D, Bates J, et al. Identifying putative candidate genes and pathways involved in immune responses to porcine reproductive and respiratory syndrome virus (PRRSV) infection. Anim Genet. 2012;43(3):328-32.

22. Arceo ME, Ernst CW, Lunney JK, Choi I, Raney NE, Huang T, et al. Characterizing differential individual response to porcine reproductive and respiratory syndrome virus infection through statistical and functional analysis of gene expression. Front Genet. 2012;3:321.

23. Steibel JP, Wysocki M, Lunney JK, Ramos AM, Hu ZL, Rothschild MF, et al. Assessment of the swine protein-annotated oligonucleotide microarray. Anim Genet. 2009;40(6):883-93.

24. Langfelder P, Horvath S. WGCNA: an R package for weighted correlation network analysis. BMC Bioinformatics. 2008;9:559.

25. Reverter A, Chan EK. Combining partial correlation and an information theory approach to the reversed engineering of gene co-expression networks. Bioinformatics. 2008:24(21):2491-7.
26. Rowland RR, Lunney J, Dekkers J. Control of porcine reproductive and respiratory syndrome (PRRS) through genetic improvements in disease resistance and tolerance. Front Genet. 2012;3:260.

27. Steibel JP, Rosa GJ. On reference designs for microarray experiments. Stat Appl Genet Mol Biol. 2005;4:Article36.

28. Ritchie ME, Silver J, Oshlack A, Holmes M, Diyagama D, Holloway A, et al. A comparison of background correction methods for two-colour microarrays. Bioinformatics. 2007;23(20):2700-7.

29. Yang YH, Dudoit S, Luu P, Lin DM, Peng V, Ngai J, et al. Normalization for CDNA microarray data: a robust composite method addressing single and multiple slide systematic variation. Nucleic Acids Res. 2002;30(4):e15.

30. R Development Core Team. R: A lanuage and environment for statistical comupting. Vienna, Austria: R Foundation for Statistical Computing; 2010.

31. Smyth GK. Limma: linear models for microarray data. In: Gentleman R, Carey V, Dudoit S, Irizarry R, Huber W, editors. Bioinformatics and Computational Biology Solutions using $\mathrm{R}$ and Bioconductor. New York, NY: Springer; 2005. p. 397-420.

32. Rosa GJ, Steibel JP, Tempelman RJ. Reassessing design and analysis of two-colour microarray experiments using mixed effects models. Comp Funct Genomics. 2005;6(3):123-31.

33. Storey JD. The positive false discovery rate: A Bayesian interpretation and the q-value. Ann Stat. 2003;31(6):2013-35.

34. Eisley C, Fritz-Waters E, Choi I, Koltes J, Boddicker N, Reecy J, Lunney J, Carpenter S, Tuggle C, Liu P, Dekkers J: Analysis of gene expression in a region associated with host response to porcine reproductive and respiratory syndrome virus challenge. In: Plant \& Animal Genome XXII, San Diego, CA; 2014 https://pagconfexcom/pag/xxii/webprogram/Paper10997html.

35. Koltes JE, Fritz-Water E, Eisley CJ, Choi I, Bao H, Kommadath A, et al. Identification of a putative quantitative trait nucleotide in Guanylate Binding Protein 5 for host response to PRRS virus infection. BMC Genomics. 2015;16:412.

36. Langfelder P, Horvath S. Fast R Functions for Robust Correlations and Hierarchical Clustering. J Stat Softw. 2012;46(11):i11.

37. Langfelder $\mathrm{P}$, Horvath $\mathrm{S}$. Eigengene networks for studying the relationships between co-expression modules. BMC Syst Biol. 2007;1:54

38. Shoemaker JE, Lopes TJ, Ghosh S, Matsuoka Y, Kawaoka Y, Kitano H. CTen: a web-based platform for identifying enriched cell types from heterogeneous microarray data. BMC Genomics. 2012;13:460.

39. Koesterke L, Milfeld K, Vaughn M, Stanzione D, Koltes J, Weeks N, et al. Optimizing the PCIT algorithm on stampede's Xeon and Xeon Phi processors for faster discovery of biological networks. In: Proceedings of the Conference on Extreme Science and Engineering Discovery Environment: Gateway to Discovery. 2013. p. 2013.

40. Koesterke L, Koltes J, Weeks N, Milfeld K, Vaughn M, Reecy J, et al. Discovery of biological networks using an optimized partial correlation coefficient with information theory algorthim on Stampede's Xeon and Xeons Phi processors. Concurrency Computation Prac Experience. 2014;26:2178-90.

41. da Huang W, Sherman BT, Lempicki RA. Systematic and integrative analysis of large gene lists using DAVID bioinformatics resources. Nat Protoc. 2009;4(1):44-57.

42. Freeman TC, Goldovsky L, Brosch $M$, van Dongen S, Maziere $P$, Grocock RJ, et al. Construction, visualisation, and clustering of transcription networks from microarray expression data. PLoS Comput Biol. 2007;3(10):2032-42.

43. Liu S, Lin L, Jiang P, Wang D, Xing Y. A comparison of RNA-Seq and high-density exon array for detecting differential gene expression between closely related species. Nucleic Acids Res. 2011;39(2):578-88.

44. Sirbu A, Kerr G, Crane M, Ruskin HJ. RNA-Seq vs dual- and single-channel microarray data: sensitivity analysis for differential expression and clustering. PLoS One. 2012;7(12):e50986.

45. Zhao S, Fung-Leung WP, Bittner A, Ngo K, Liu X. Comparison of RNA-Seq and microarray in transcriptome profiling of activated T cells. PLoS One. 2014;9(1):e78644

46. Fuller TF, Ghazalpour A, Aten JE, Drake TA, Lusis AJ, Horvath S. Weighted gene coexpression network analysis strategies applied to mouse weight. Mamm Genome. 2007;18(6-7):463-72.

47. Van Gucht S, Van Reeth K, Nauwynck H, Pensaert M. Porcine reproductive and respiratory syndrome virus infection increases CD14 expression and lipopolysaccharide-binding protein in the lungs of pigs. Viral Immunol. 2005;18(1):116-26 
48. Costers S, Lefebvre DJ, Delputte PL, Nauwynck HJ. Porcine reproductive and respiratory syndrome virus modulates apoptosis during replication in alveolar macrophages. Arch Virol. 2008;153(8):1453-65.

49. Teodoro JG, Branton PE. Regulation of apoptosis by viral gene products. J Virol. 1997;71(3):1739-46.

50. Takeuchi O, Akira S. Pattern recognition receptors and inflammation. Cell. 2010;140(6):805-20.

51. Schoggins JW, Rice CM. Interferon-stimulated genes and their antiviral effector functions. Curr Opin Virol. 2011;1(6):519-25.

52. Dawson HD, Loveland JE, Pascal G, Gilbert JG, Uenishi H, Mann KM, et al. Structural and functional annotation of the porcine immunome. BMC Genomics. 2013;14:332.

53. Miller LC, Laegreid WW, Bono JL, Chitko-McKown CG, Fox JM. Interferon type I response in porcine reproductive and respiratory syndrome virus-infected MARC-145 cells. Arch Virol. 2004;149(12):2453-63.

54. Van Reeth K, Labarque G, Nauwynck H, Pensaert M. Differential production of proinflammatory cytokines in the pig lung during different respiratory virus infections: correlations with pathogenicity. Res Vet Sci. 1999;67(1):47-52.

55. Souza C, Choi I, Araujo K, Abrams S, Kerrigan M, Rowland RR, Lunney J: Comparative serum immune responses of pigs after a challenge with porcine reproductive and respiratory syndrome virus (PRRSV). In: Proceedings of the 10th IVIS International Veterinary Immunology Symposium, P0514: 2013; Milan, Italy; 2013, www.ivis2013.org/allegati/ Abstract_book.pdf.

56. Hulst M, Loeffen W, Weesendorp E. Pathway analysis in blood cells of pigs infected with classical swine fever virus: comparison of pigs that develop a chronic form of infection or recover. Arch Virol. 2013;158(2):325-39.

57. Rauw WM. Immune response from a resource allocation perspective. Front Genet. 2012;3:267.

58. Tschopp J, Schroder K. NLRP3 inflammasome activation: The convergence of multiple signalling pathways on ROS production? Nat Rev Immunol. 2010;10(3):210-5

59. Bi J, Song $\mathrm{S}$, Fang $L$, Wang $D$, Jing $H$, Gao L, et al. Porcine reproductive and respiratory syndrome virus induces IL-1 beta production depending on TLR4/MyD88 pathway and NLRP3 inflammasome in primary porcine alveolar macrophages. Mediators Inflamm. 2014;2014:403515.

60. Lee CC, Freinkman E, Sabatini DM, Ploegh HL. The protein synthesis inhibitor blasticidin s enters mammalian cells via leucine-rich repeat-containing protein 8D. J Biol Chem. 2014;289(24):17124-31.

61. Pilla DM, Hagar JA, Haldar AK, Mason AK, Degrandi D, Pfeffer K, et al. Guanylate binding proteins promote caspase-11-dependent pyroptosis in response to cytoplasmic LPS. Proc Natl Acad Sci U S A. 2014;111(16):6046-51.

62. Wagner E, Frank MM. Therapeutic potential of complement modulation. Nat Rev Drug Discov. 2010;9(1):43-56.

\section{Submit your next manuscript to BioMed Central and take full advantage of:}

- Convenient online submission

- Thorough peer review

- No space constraints or color figure charges

- Immediate publication on acceptance

- Inclusion in PubMed, CAS, Scopus and Google Scholar

- Research which is freely available for redistribution 